\title{
Mesure de température et de flux de chaleur à base de couches minces ou épaisses: atouts, fabrication et applications
}

\author{
Bertrand Garnier ${ }^{1, a}$ \\ ${ }^{1}$ Laboratoire de Thermocinétique, UMR CNRS 6607, Polytech'Nantes, 44306 Nantes cdx 3, France
}

\begin{abstract}
Temperature measurements on surfaces or inside mediums are often performed using resistance temperature detectors (RTD) or thermocouple wires. The wire thermocouples are preferred for transient measurement because they are relatively less intrusive than other types of sensors and have low response times until a few tens of milliseconds. The smallest diameters of thermocouple wires are of the order of $12 \mu \mathrm{m}$, however with such a size they are very difficult to implement. In addition, there are many situations in which theirs dimensions are still too large as in microsystems such as OLED, organic solar cell, hybrid thermoelectric devices and also microsensors.... Thus, it is very advantageous to use temperature sensors as thin films where the thickness is typically of the order of $100 \mathrm{~nm}$ and also thick film (on the order of a few micrometers). In this communication, we present successively: i) the advantages and characteristics of thermal metrology using thin or thick films, ii) their design and manufacturing, iii) the specificities of thin film thermocouples and resistance thermometers and finally various examples of achievement.
\end{abstract}

\section{Contexte et objectifs}

La métrologie a un rôle important car elle intervient pour prendre des décisions que ce soit pour l'acquisition ou la vérification de produits lors de la comparaison de leurs performances ou pour des actions de recherche afin de valider ou non des hypothèses ou des modèles théoriques.

Dans le domaine de l'énergie, les mesures de température sont essentielles pour caractériser les performances énergétiques des produits ou de systèmes. Cependant les mesures de température avec des procédés par contact sont très souvent réalisées par des thermorésistances ou des thermocouples filaires. Les thermocouples filaires sont préférables car ils sont relativement peu intrusifs et ont de faibles temps de réponse. Les plus petits diamètres de fils sont de l'ordre de $12 \mu \mathrm{m}$. Cependant avec une telle taille, ils sont très difficiles à mettre en place. De plus, il y a de nombreuses situations pour lesquelles ces dimensions sont encore trop importantes (microréacteurs, microsystèmes, microcapteurs...). Il est alors très intéressant d'utiliser des capteurs sous forme de couches épaisses (de l'ordre de quelques $\mu \mathrm{m}$ ) ou mieux de couches minces où l'épaisseur est typiquement de l'ordre de $100 \mathrm{~nm}$.
Les applications des capteurs de température réalisés à base de couches minces sont nombreuses [1]. Cela concerne les phénomènes rapides comme la caractérisation des propriétés thermiques de couches submicroniques pour des applications de type barrière thermique [2] ou pour la dissipation de la chaleur dans des dispositifs électroniques [3].

Les capteurs couches minces sont également utilisés dans les dispositifs pour les mesures fluxmétriques très précises ou spatialement résolues (bolomètre [4]). Par ailleurs, il est possible de réaliser des thermocouples avec des couches transparentes pour des mesures de température sur des dispositifs optiques afin de limiter les distorsions (télescope [5]) ou les surchauffes (optique pour laser [6]).

Enfin dans de nombreux secteurs industriels, on utilise de la métrologie thermique à base de couches minces. On peut citer le domaine de la plasturgie avec des capteurs d'évènements thermiques [7] ou dans des dispositifs fonctionnant à température élevée. Pour ces derniers, l'obtention de la stabilité en température des propriétés électriques des dépôts ainsi qu'un faible de temps réponse a conduit à des applications à haute température sur des aubes de turbines ou dans des moteurs à combustion interne $[8,9]$.

\footnotetext{
a bertrand.garnier@univ-nantes.fr
} 
Dans cette communication, on présentera successivement

-les avantages et les spécificités de la métrologie par couche mince ou épaisse,

-la conception et la fabrication des dépôts,

-la spécificité des thermocouples et résistances thermométriques sous forme de couche mince et,

- quelques exemples de réalisation et d'utilisation.

\section{Avantage et spécificités des couches minces ou épaisses}

Des couples thermoélectriques et des résistances thermométriques peuvent être déposés sur la surface sous forme de films très minces de quelques dizaines à centaines de nanomètres d'épaisseur (couches minces) ou de quelques micromètres à plusieurs dizaines de $\mu \mathrm{m}$ d'épaisseur (couches épaisses).

Les avantages de l'utilisation des couches minces ou épaisses en métrologie thermique sont multiples : les biais de mesure dus à leur mise en place dans les dispositifs étudiés sont nettement moins importants qu'avec des thermocouples filaires; en effet les pertes thermiques à travers le capteur sont nettement moindres. En couche mince, les temps de réponse des capteurs sont évidemment plus faibles. Ainsi les temps de réponse sont typiquement de l'ordre de $10^{-9}$ à quelques $10^{-6}$ secondes. Contrairement aux capteurs filaires, les capteurs de type couches minces déposés sur une surface ont une meilleure qualité de liaison avec celle-ci (résistance de contact bien plus faible).

Par ailleurs, les techniques de fabrication des couches minces permettent de réaliser simultanément plusieurs capteurs et on peut lors des dépôts intégrer également des actionneurs. Du point de vue pratique, l'usage de couches minces pour des mesures de température nécessite de prendre des précautions. Par exemple les pouvoirs thermoélectriques des matériaux déposés en couches minces peuvent dépendre de l'épaisseur du dépôt et un effet thermoélectrique peut apparaître lorsque les fils de connexion pourtant de même nature sont raccordés au dépôt.

\section{Conception et fabrication des dépôts de couches minces et épaisses}

\subsection{Choix des matériaux à déposer}

On peut déposer plusieurs types de matériaux ceux-ci ayant des fonctions différentes. On peut citer le dépôt de conducteurs (métaux purs, alliages, semi-conducteurs...), de diélectriques (silice, alumine...), de couches d'accrochage (Cr, Ni, CuNi, NiCr...), de dispositifs de connexion électrique (Au, AuPt, AuSn pour assurer la liaison fil/films par soudure) et enfin de barrière de protection pour empêcher la formation de composés d'interdiffusion notamment à température élevée.

\subsection{Fabrication de couches minces}

Les couches minces sont réalisées par des procédés de dépôts sous vide (évaporation et sublimation thermique, pulvérisation cathodique...).

Les motifs sont obtenus à l'aide de masques. Par électroérosion, on peut réaliser des masques métalliques qui permettent d'obtenir des pistes déposées avec des largeurs supérieures à $100 \mu \mathrm{m}$. En utilisant un laser, on peut obtenir des masques permettant de produire des pistes avec des largeurs de seulement $10 \mu \mathrm{m}$ [10]. La photolithogravure permet d'atteindre quelques microns. D'excellents états de surface sont nécessaires afin d'obtenir la continuité électrique des dépôts. Il faut noter que la nature du ou des matériaux choisis ainsi que du procédé de fabrication affecte les propriétés physiques de la couche déposée et notamment ses propriétés électriques. Pour la couche conductrice ou isolante, on souhaitera une conductivité électrique adéquate, une bonne adhésion avec les couches voisines ou le substrat ainsi qu'une certaine dureté...

\subsection{Fabrication de couches épaisses}

Des procédés plus simples peuvent être mis en œuvre : attaque de dépôts électrolytiques, utilisation de peintures sous forme d'aérosol, sérigraphie. Cependant il en résultera des épaisseurs plus importantes de une à quelques dizaines ou centaines de micromètres ainsi que des performances moindres (temps de réponse, stabilité en température) mais parfois suffisantes pour le problème de métrologie abordé. Des exemples d'usage des deux premiers procédés sont présentés dans la section 5. Le troisième procédé de réalisation de couches épaisses qui est la sérigraphie est très courant. Dans celui-ci, l'encre sérigraphiable comporte une phase minérale et une phase organique dont les solvants les plus volatiles disparaissent au cours de l'étuvage autour de $120^{\circ} \mathrm{C}$ et la phase solide organique disparaît par combustion ou décomposition lors du traitement thermique entre 350 et $750^{\circ} \mathrm{C}$. C'est la phase minérale qui confère la propriété électrique souhaitée au dépôt. La largeur minimale des pistes conductrices sérigraphiées est de $100 \mu \mathrm{m}$.

\section{Spécificités liées aux phénomènes thermométriques}

\subsection{Couples thermoélectriques}

A priori tous les couples thermoélectriques standards conviennent; cependant on préfère les métaux purs [11] car la reproductibilité de la composition des alliages est plus difficile à obtenir lors de la réalisation des dépôts.

Un étalonnage in situ est souvent nécessaire du fait de la forte dépendance des pouvoirs thermoélectriques aux conditions d'élaboration et à la structure physicochimique du substrat (nature du matériau, microrugosité). En effet, 
les structures souvent colonnaires et la présence d'impuretés conduisent à des pouvoirs thermoélectriques plus faibles que ceux des matériaux massifs (Tableau 1).

Tableau 1. Pouvoir thermoélectrique du constanstan $(0,4$ Ni 0,6 $\mathrm{Cu}$ ) évaporé sous vide sur du quartz

\begin{tabular}{|c|l|l|l|l|}
\hline Epaisseur $(\mathrm{nm})$ & 40 & 100 & 250 & Massif \\
\hline $\mathrm{PT}^{*}(\mu \mathrm{V} / \mathrm{K})$ & -30 & -34 & -38 & -42 \\
\hline
\end{tabular}

*: PT : pouvoir thermoélectrique à $20^{\circ} \mathrm{C}$

Hormis la nécessité de réaliser leur étalonnage, un des inconvénients de la dépendance du pouvoir thermoélectrique avec l'épaisseur du dépôt est l'apparition d'un thermocouple parasite à la connexion entre couche mince et fil de connexion même s'ils sont tous les deux réalisés avec le même type de métal ou d'alliage.

\subsection{Résistances thermométriques}

Les capteurs de type résistance thermométrique utilisent la dépendance en température de la résistivité électrique des métaux ou des alliages comme phénomène thermométrique. Dans les versions à base de couches minces ou épaisses, il est préférable de réaliser l'étalonnage pour chaque nouveau type et conditions de dépôt en effet les conductivités électriques et les sensibilités sont fortement dépendantes de la morphologie $\mathrm{du}$ dépôt (impuretés, défauts structuraux, limitation $\mathrm{du}$ libre parcours moyen des porteurs de charge ...). Pour réduire ces effets, les métaux purs sont à privilégier. Il est à noter que le coefficient de température $\alpha_{R}$ de la résistivité électrique des couches minces submicroniques est inférieur à celui des matériaux massifs (Tableau 2). Par ailleurs dans le même tableau, il apparait que si en version massif le nickel est préférable en terme de sensibilité, ce n'est plus le cas en version couche mince réalisée par évaporation.

En détectant les variations de différence de potentiel par un montage potentiométrique (simple ou double -i.e. pont de Wheatstone), on peut obtenir des sensibilités de l'ordre de quelques dixièmes à quelques $\mathrm{mV} /{ }^{\circ} \mathrm{C}$.

Tableau 2. Dépendance en température du coefficient de température $\alpha_{R}$ de la résistivité électrique pour des couches minces déposées par évaporation d'or, de nickel, d'aluminium et de chrome [12] et valeur pour le matériau massif

\begin{tabular}{|c|c|c|c|c|}
\hline $\begin{array}{c}\alpha_{R} \\
/\left(10^{-3} \mathrm{~K}^{-1}\right)\end{array}$ & $\begin{array}{c}\mathrm{Au}(200 \mathrm{n}) \\
/ \mathrm{Cr}(20 \mathrm{~nm})\end{array}$ & $\begin{array}{c}\mathrm{Ni} \\
(400 \mathrm{~nm})\end{array}$ & $\begin{array}{c}\mathrm{Al} \\
(220 \mathrm{~nm})\end{array}$ & $\begin{array}{c}\mathrm{Cr} \\
(162 \mathrm{~nm})\end{array}$ \\
\hline $300 \mathrm{~K}$ & 2.7 & 2.3 & 1.4 & 0.17 \\
\hline $350 \mathrm{~K}$ & 2.4 & 2.2 & 1.3 & 0.17 \\
\hline
\end{tabular}

\begin{tabular}{|c|c|c|c|c|}
\hline $\begin{array}{c}\text { Massif } \\
(300 \mathrm{~K})\end{array}$ & 4.0 & 6.7 & 4.5 & 2.1 \\
\hline
\end{tabular}

Par exemple, Sommier [13] a obtenu une sensibilité de $20 \mathrm{mV} / \mathrm{K}$, c'est à dire 500 fois la sensibilité d'un thermocouple de type K. Ainsi, une forte sensibilité peut être obtenue en augmentant le courant dans la résistance thermométrique, cependant il faut être prudent car cela se traduit par un auto-échauffement du dépôt par effet Joule engendrant des biais de mesure de température. Ainsi en pratique, le courant sera limité à quelques mA. La Figure 1 illustre un exemple de calcul d'auto échauffement d'un dépôt d'or d'épaisseur $100 \mathrm{~nm}$, de largeur $30 \mu \mathrm{m}$ et de longueur $500 \mu \mathrm{m}$ destiné à caractériser l'élévation de température dans un microréacteur. Le dépôt étant en contact avec l'écoulement des réactifs, l'auto-échauffement est dépendant du coefficient d'échange fluides réactifs / paroi. Une modélisation thermique et électrique a permis de chiffrer l'auto échauffement à moins de $0,1{ }^{\circ} \mathrm{C}$ si l'échange est assez important (coefficient d'échange $h$ supérieur à $5000 \mathrm{~W} \cdot \mathrm{m}^{-2} \cdot \mathrm{K}^{-1}$ ). Les perturbations en terme de flux de chaleur et de température dues à l'effet Joule restent donc faibles dans les thermorésistances pour le courant d'alimentation utilisé (4,5 mA).
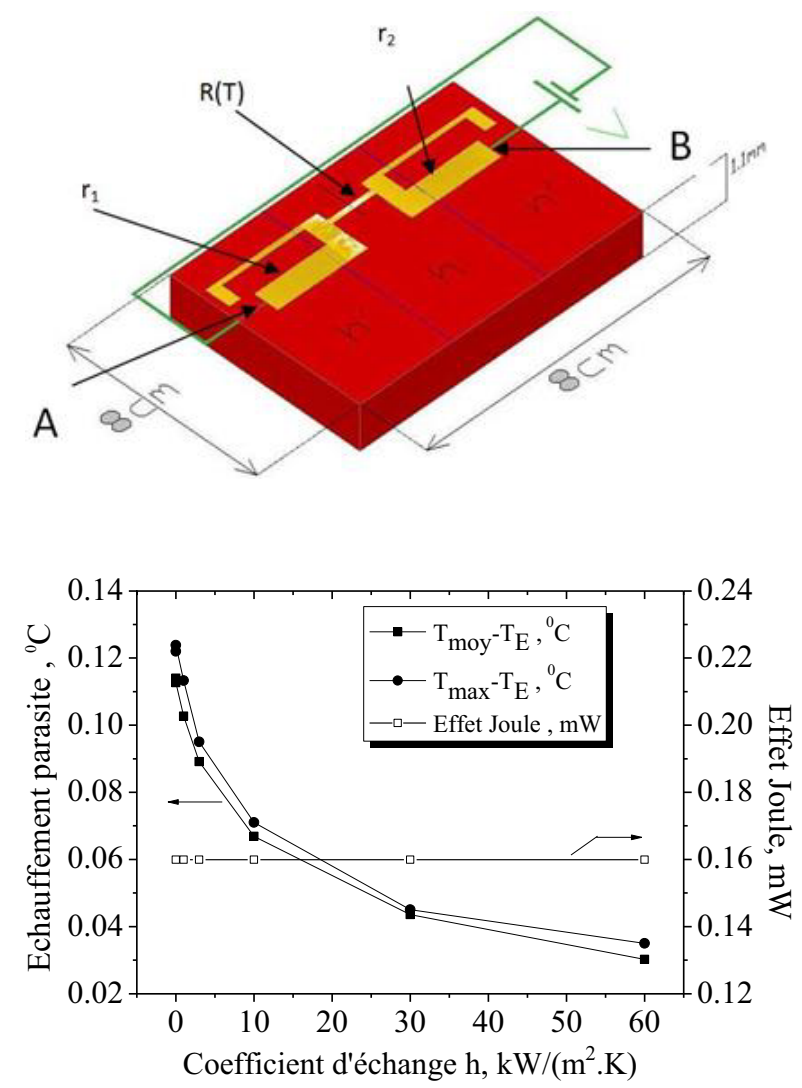
Figure 1. a) Modèle électrothermique étudié et b) autoéchauffement de $\mathrm{R}(\mathrm{T})$ en fonction du coefficient d'échange dans le microcanal $[13]$ ( $\mathrm{T}_{\mathrm{E}}$ : température initiale)

On rencontre les capteurs de type résistance thermométrique sous forme de couches minces ou épaisses notamment lorsque de très faibles dimensions de celui-ci sont requises ou lors de mesures de très faibles flux de chaleur. On peut citer par exemple la cartographie de l'univers à l'aide de détecteurs bolométriques (sensibilité jusqu'à $10^{-16} \mathrm{~W}$ [5]). On trouvera également de nombreuses applications en laboratoire (microréacteur, caractérisation de couches minces...) là où de très faibles dimensions des capteurs sont requises de part la faible taille du microsystème à étudier.

\section{Quelques exemples d'utilisation de couches thermométriques minces ou épaisses}

Dans ce qui suit, on présente divers exemples où l'utilisation des couches minces est particulièrement pertinente. Ces applications concernent le domaine des microréacteurs, des capteurs thermiques ou des dispositifs industriels nécessitant une micro-instrumentation nettement moins intrusive qu'avec des microthermocouples filaires et là où les techniques par caméra infrarouge ne permettent pas les mesures de champ de température en raison de la non accessibilité des surfaces à analyser.

Le domaine de la microfluidique et des microréacteurs est en plein essor. Lorsque les microcanaux sont sièges de transferts de chaleur (microéchangeur, microréacteur) l'instrumentation avec capteurs en paroi leur confère une grande sensibilité aux évènements thermiques dans l'écoulement. L'utilisation de résistances thermométriques en or a ainsi permis de valider les corrélations macroscopiques $\mathrm{Nu}=\mathrm{Nu}(\mathrm{Re}, \mathrm{Pr})$ dans des microcanaux jusqu'à une hauteur de seulement $50 \mu \mathrm{m}$ (Figure 2 [14]).

Des techniques de lithographie et par évaporation ont été utilisées. Un dispositif de même type a permis d'étudier les effets thermiques d'une réaction de neutralisation acide/base ainsi que l'effet d'un procédé actif de mélange [15].

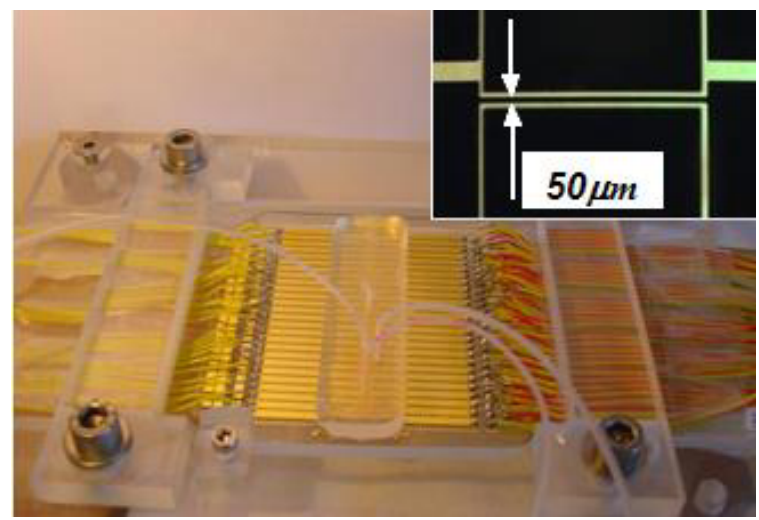

Figure 2. Fluxmètres 2D pour la microfluidique avec 25 résistances thermométriques en or déposées de part et d'autre d'un substrat de borosilicate [14].

La Figure 3 illustre un exemple de capteur fluxmétrique à installer dans une paroi permettant des mesures précises de flux de chaleur, celui-ci a été réalisé par attaque chimique de feuille de polymère recouverte de cuivre d'épaisseur $9 \mu \mathrm{m}$ [16] réalisé par le fournisseur à l'aide d'un procédé de cuivrage chimique autocatalytique. Un étamage à froid permet d'empêcher l'oxydation. Ces dépôts sur feuille de polyimide (kapton) ou de plaque de résine époxy étant facilement disponible, la technique de réalisation du fluxmètre est simple et peu coûteuse.

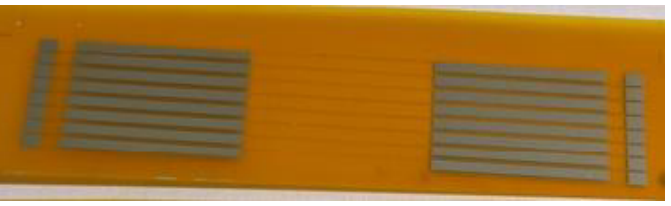

a)

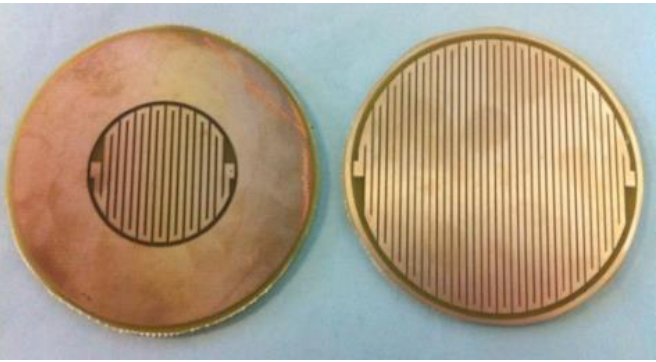

Figure 3. Fluxmètres thermiques 1D a) passif sur feuille de kapton et b) dynamique tous les deux réalisés par attaque chimique de dépots de cuivre suivi d'un étamage à froid [14].
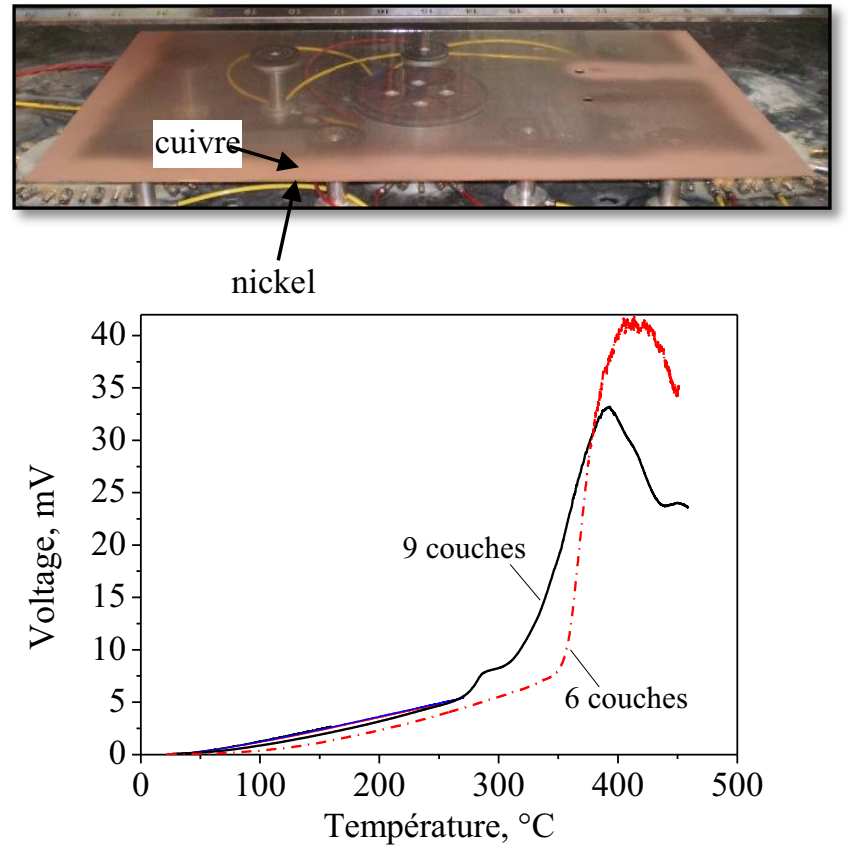

Figure 4. a) Dépôts pulvérisés de cuivre et de nickel pour la mesure 
de température sur le flan d'une paroi de verre. b) étalonnage du thermocouple cuivre - nickel pulvérisé en fonction du nombre de couches.

Lors de la réalisation des couches minces, les dimensions des capteurs sont limitées par les dimensions admissibles de substrat dans les bâtis de dépôt sous vide. Sur des objets industriels de grande dimension (plaque de verre [17], Figure 4), il est alors possible d'utiliser des peintures aérosols, ceux de type $\mathrm{Cu}$ et $\mathrm{Ni}$ étant facilement disponibles. Avec les épaisseurs obtenues entre 20 et $30 \mu \mathrm{m}$, on retrouve la sensibilité en temperature du thermocouple massif $\mathrm{Cu}-\mathrm{Ni}$. Cependant, l'usage à des températures élevées est à proscrire en raison de l'oxydation très rapide du cuivre induisant une forte modification de la réponse du thermocouple comme l'illustre la Figure $4 \mathrm{~b}$ à partir de $250^{\circ} \mathrm{C}$, un recuit est de toute manière recommandé pour stabiliser les pouvoirs thermoélectriques.

\section{Conclusion}

La miniaturisation de dispositifs électroniques, fluidiques et la nécessité de mesures très localisées induit un besoin d'utilisation de métrologie très peu intrusive. A ces échelles en thermique, les résistances thermométriques et les thermocouples filaires ne sont plus adaptés, d'autant plus que pour ces derniers dans la plus faible gamme de diamètre existant $(0,5-25 \mu \mathrm{m})$, ils sont particulièrement difficiles à implémenter. L'usage de capteurs de température sous forme de couches déposées est alors une solution à privilégier. Cependant, il faut veiller lors de la conception de ces capteurs à limiter les biais de mesure liés à la spécificité des couches minces ou épaisses (pouvoir thermoélectrique différent du massif, sensibilité à l'oxydation et aux phénomènes de diffusion notamment à température élevée). Par ailleurs, la réalisation de dépôts à l'aide de procédés sous vide reste une opération complexe en raison des nombreuses étapes de fabrication, cependant il existe des procédés plus simples (attaque chimique de dépôts électrolytiques, sérigraphie, utilisation de peintures aérosols...) permettant de réaliser plus simplement des couches certes plus épaisses mais moins coûteuses à fabriquer.

\section{Références}

1. J.P. Prenei, F. Lanzetta, E. Gavignet, Y. Bailly, B. Serio, P. Nika, L. Thiery Thermal microsensors: applications to temperature and fluidic measurements in Encyclopedia of Sensors ed. C.A. Grimes, E.C. Dickey, M.V. Pishkovol 10 (Am. Sci. Pub. 2006) 217245

2. D. Burgess, M. Yust, K.G. Kreider, Sens Actuators A24 (1990) 155-161.

3. M. Bogner, A. Hofer, G. Benstetter, H. Gruber, R.Y.Q. $\mathrm{Fu}$, Thin Solid Films (2015) in press
4. B. Lakew, S. Aslam, J. Brasunas, N. Cao, N. Costen, A. La, L. Nguyen, T. Stevenson, A. Waczynski Physica C: Superconductivity 483 (2012)119-126

5. http://www.herschel.fr/fr/telescope_et_instruments/ les_bolometres.php

6. K.G. Kreider, Sens. Actuators A34 (1992) 95-99

7. A. Ferrec, C. Plot, N. Allanic, P.Y. Jouan, A. Sarda, P. Mousseau, Actes du Congrès Français de Thermique SFT, pp. 1-8, La Rochelle 26-29 mai 2015

8. G.E. Aniolek, O.J. Gregory, Surf. Coat. Technol. 68/69 (1994) 70-75

9. K.G. Kreider, J. Vac. Sci. Technol. A4 (1986) 26182623

10. F. Reisdorffer, B. Garnier, N. Horny, C. Renaud, M. Chirtoc, T.P. Nguyen, EPJ, 79 (2014) 02001

11. X. Tian, F.E. Kennedy, J.J. Deacutis, A.K. Henning, Tribol. Trans., 35 3(1992) 491-499

12. A. Jacquot, Ingénierie des Matériaux et des Microgénérateurs Thermoélectriques Planaires, Thèse Inst. Nat. Polyt. Lorraine, 28 mars 2003.

13. B. Garnier, A. Sommier., J. Reinf. Plastics Comp., 21 13 (2002)1193-1203

14. D. Hamadi, B. Garnier., H. Willaime., F. Monti, H. Peerhossaini, Lab On a Chip, 12 (2012) 652-658

15. H. Ammar' B. Garnier, A. Ould El Moctar, F. Monti, H. Willaime, K. Loubiere K., L. Prat, C. Gourdon, H. Peerhossaini, Actes du Congrès Français de Thermique SFT, pp. 1-6, Perpignan 24 au 27 mai 2011.

16. B. Azerou, B. Garnier, J. Lahmar, J. Phys. CS 395 (2012) 012084

17. T. Moussa, Refroidissement du point de contact meule/verre plat par un échangeur multi-jets lors $d u$ façonnage, Thèse Univ. de Nantes, 10 décembre 2013. 